\title{
Improved visualisation of a coronary artery fistula by the "laid-back" aortogram
}

\author{
Michael Hofbeck, Florian Wild, Helmut Singer
}

Department of Paediatric Cardiology University Children's Hospital, Erlangen, Germany M Hofbeck F Wild H Singer

Correspondence to: Dr Michael Hofbeck Loschgestrasse 15 Universitätskinderklinik D-8520 Erlangen, Germany.

\begin{abstract}
The preoperative assessment of patients with a coronary artery fistula requires exact angiographic evaluation of the coronary artery anatomy. A technique of transvenous balloon occlusion angiography of the aorta is described. This can be applied easily in infants with a persisting foramen ovale. Combination of this technique with the so-called "laid-back" aortogram gave excellent visualisation of a fistula between the right coronary artery and the right ventricle. This technique is a major improvement in the angiographic assessment of infants with coronary artery abnormalities and normal cardiac connections.
\end{abstract}

(Br Heart f 1993;70:272-273)

Balloon occlusion angiography of the aortic root has become routine for the preoperative assessment of neonates with d-transposition

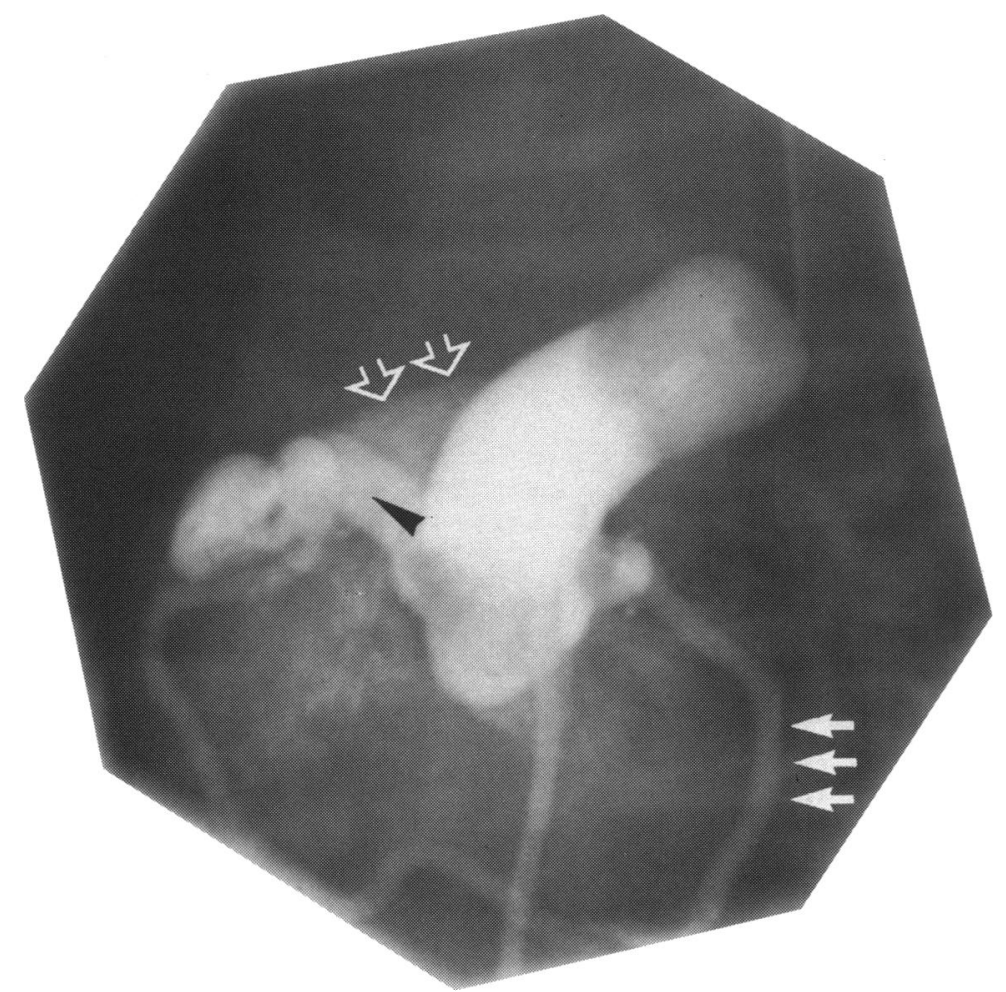

Figure 1 Balloon occlusion aortogram (left anterior oblique projection) showing an enlarged right coronary artery (black arrow) with subsequent opacification of the right ventricular outflow tract (double arrow). The triple arrow indicates the left circumflex artery. of the great arteries. ${ }^{1}$ Demonstration of the coronary artery anatomy can be optimised by the application of the so-called "laid-back" aortogram. ${ }^{2}$ This projection is obtained by a $40-45^{\circ}$ caudal tilt of the frontal camera. A slight left or right anterior oblique orientation may be added to foreshorten the ascending aorta. In neonates and young infants a persisting foramen ovale allows anterograde catheterisation of the left heart. We report balloon occlusion angiography of the aorta from a transvenous approach in a patient with normal cardiac connections.

\section{Case report}

Echocardiography when this patient was three weeks old showed a fistula from the right coronary artery to the right ventricle. Cardiac catheterisation was performed a week later. Access was gained percutaneously through the right femoral vein. The foramen ovale was crossed with a 5 French balloon angiographic catheter (American Edwards Laboratories, Swan-Ganz). The catheter was advanced via the left ventricle into the ascending aorta and was positioned proximally to the innominate artery, so that the sideholes were $1 \mathrm{~cm}$ above the aortic valve. With temporary occlusion of the aorta we performed two power injections for cineangiography using biplane projections. Angiography was performed first in the anteroposterior and lateral projection and then with a $45^{\circ}$ caudal tilt of the frontal $x$ ray tube which added a $5^{\circ}$ left anterior oblique orientation. The lateral tube was positioned with a $30^{\circ}$ left anterior oblique orientation. We injected 1.5 and $2.0 \mathrm{ml} / \mathrm{kg}$ of non-ionic contrast medium (Ultravist 370) in $1 \mathrm{~s}$. The laid-back aortogram gave the best information on the origin of the coronary artery fistula and its communication with the right ventricle (figs 1 and 2).

\section{Discussion}

Coronary artery fistulas can occur between the coronary arteries and the cardiac chambers, coronary sinus, superior vena cava, or pulmonary arteries. ${ }^{1}$ High quality angiographic demonstration of the coronary artery anatomy usually requires retrograde aortography or selective coronary angiography with arterial access. Arterial catheterisation of 


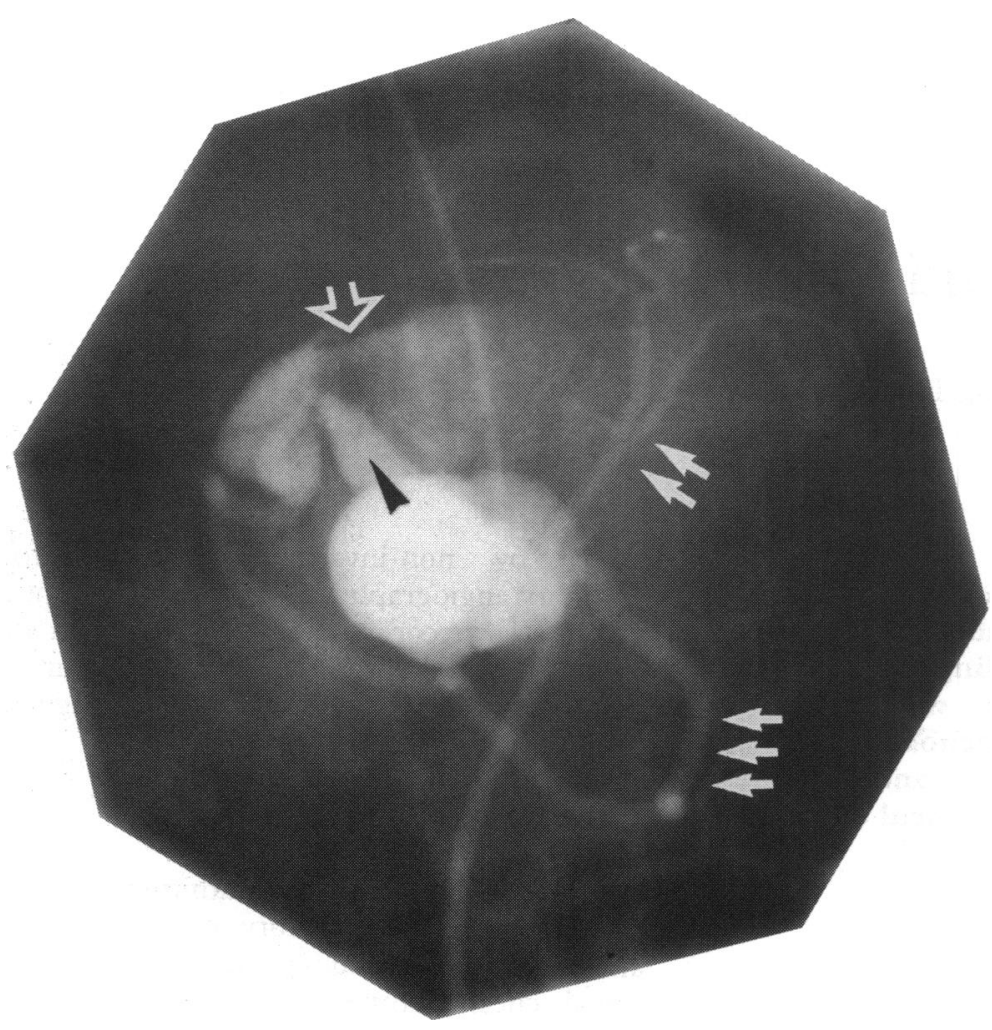

Figure 2 Balloon occlusion aortogram in the laid-back view showing a dilated and tortuous right coronary artery (black arrow). Contrast medium passed through the fistula into the right ventricle (white arrow). The double arrow indicates the left anterior descending artery and the triple arrow the left circumflex artery. infants is often complicated by an arterial spasm or thrombus formation at the site of entry. In patients with a persisting foramen ovale or an atrial septal defect aortography can be performed by passing a balloon catheter through a vein, even when the cardiac connections are normal. ${ }^{3}$ Balloon occlusion angiography of the ascending aorta in combination with a caudal tilt of the frontal camera (the so-called laid-back aortogram) gives excellent delineation of the coronary artery anatomy. In our experience the angiographic demonstration of a coronary artery fistula requires a higher volume of contrast medium than angiographic demonstration of the coronary artery anatomy in patients with d-transposition of the great arteries. ${ }^{2}$

1 Burrows PE, Freedom RM. Anomalies of the coronary arteries. In: Freedom RM, Benson LN, Smallhorn JF, eds. Neonatal heart disease. Berlin: Springer Verlag,

2 Mandell VS, Lock JE, Mayer JE, Parness IA, Kulik TJ. The "laid-back" aortogram: An improved angiographic view for demonstration of coronary arteries in transposition of the great arteries. Am 7 Cardiol 1990;65 1379-83.

3 Burrows P, Benson LN, Freedom RM. Coronary angiography in pulmonary atresia with intact ventricular septum. In: Freedom RM, ed Pulmonary atresia with intact ventricular septum. Mount Kisco, New York: Futura Publishing Company, 1989;207-12. 